\title{
Randomised controlled trial of brief neonatal exposure to cows' milk on the development of atopy
}

Marijke H de Jong, Vera T M Scharp-van der Linden, Rob C Aalberse, Johannes Oosting, Jan G P Tijssen, Cornelis J de Groot

\begin{abstract}
Objective-To determine the effect of brief early exposure to cows' milk on atopy in the first 2 years of life.

Design-Double blind, placebo controlled, randomised feeding intervention trial (Bokaal study).

Setting-Dutch midwifery practices. Participants-1533 breast fed neonates. Intervention-Exposure to cows' milk protein $(n=758)$ or a protein free placebo $(n=775)$ during the first 3 days of life.

Main outcome measures-Clinical atopic disease and any positive radioallergosorbent (RAST) tests at 1 year of age.

Results-Atopic disease in the first year was found in $10.0 \%$ (cows' milk) $v \mathbf{9 . 3 \%}$ (placebo) of the children, with a relative risk of 1.07; in the second year, atopic disease was found in $9.6 \% v 10.2 \%$, respectively, with a relative risk of 0.94 . Per protocol analysis showed similar results. Any RAST positive test was found in $9.4 \%$ (cows' milk) $v \mathbf{7 . 9 \%}$ (placebo) of children, with a relative risk of 1.19 . Stratified analysis for high family risk of allergy showed a doubled incidence of atopic disease but no effect from the intervention.

Conclusion-Early and brief exposure to cows' milk in breast fed children does not increase the risk of atopic disease in the first 2 years.

(Arch Dis Child 1998;79:126-130)
\end{abstract}

Keywords: atopy; breast feeding; cows' milk; Bokaal study; randomised controlled trial

Breast fed infants quite often receive, sometimes inadvertently, supplementary cows' milk formula. In most cases, these feeds are given for a short period until breast milk production has been established. There is controversy about whether such cows' milk supplements influence the development of allergy at a later age. Some authors have claimed a harmful effect of early exposure, others have found no difference in the development of allergic symptoms, and a protective effect of cows' milk protein has even been suggested. Høst et al followed 1539 healthy Danish infants and found that the 39 children who developed cows' milk allergy had been exposed to this allergen in the neonatal period, whereas none of the 210 exclusively breast fed children had cows' milk allergy. ${ }^{1}$ This suggests that the early neonatal intake of foreign proteins is an important factor involved in sensitisation and subsequent allergic manifestations ("the dangerous bottle"). However, Lindfors and Enocksson found a reduced incidence of allergic symptoms at 18 months and 5 years of age in 216 "small for dates" children who received cows' milk formula before breast feeding. ${ }^{2}$ This particularly affected high risk children. Kramer designed important biological and methodological standards ${ }^{3}$ and he concluded that there had been no properly designed studies about the influence of incidental early exposure to cows' milk formula on the development of allergic symptoms in exclusively breast fed healthy children.

Therefore, to advise nursing mothers on the possible advantages and disadvantages of cows' milk supplements regarding the development of allergic symptoms, we initiated a double blind, placebo controlled, randomised study, designed to investigate the effect of brief and early exposure to cows' milk protein on the development of atopic disease in infants and young children.

\section{Study population and methods}

The study was conducted as a feeding intervention trial. Mothers of potential participants were recruited by midwives who practised in the western part and centre of the Netherlands, between February 1992 and

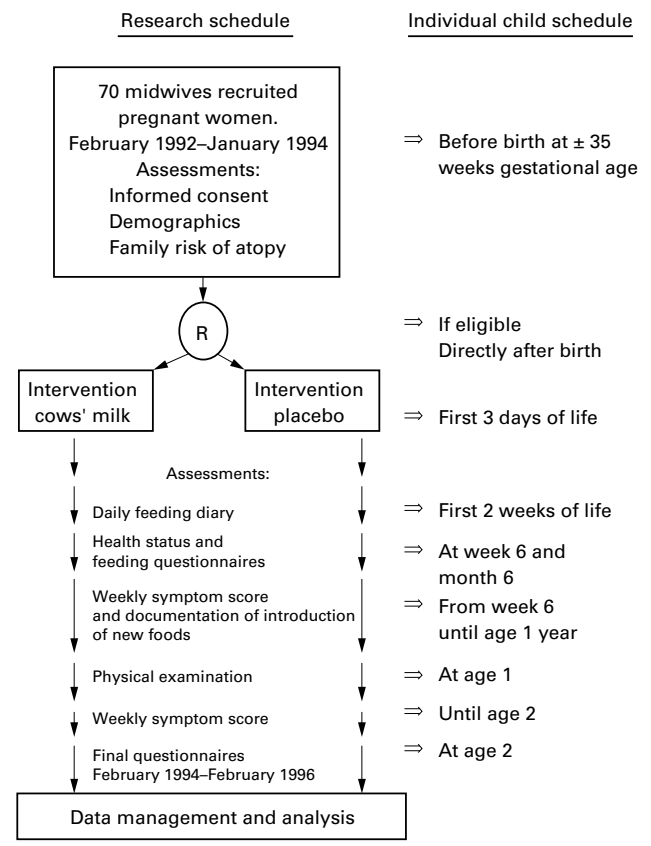

Figure 1 Study design and timetable of the Bokaal study. 


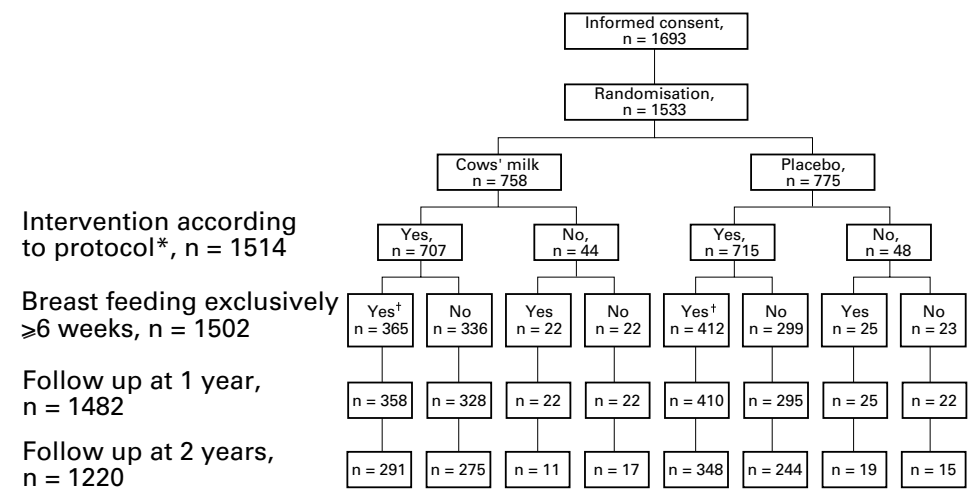

Figure 2 Study population of the Bokaal study. *, Given at least three intervention feeds; $t$, groups used for per protocol analysis.

January 1994. The protocol for the study was approved by the medical ethics committee of the Academic Medical Center of the University of Amsterdam and written informed consent was obtained from the parents before the birth of the children.

SELECTION OF THE STUDY POPULATION

Healthy, full term newborns whose mothers intended to breast feed for at least six weeks were eligible for participation in the study. Pregnant women were approached for participation by midwives, at about 35 weeks of gestation. After birth, the child entered the study if the following inclusion criteria were met: (1) parents understood the Dutch language; (2) birth weight $2750 \mathrm{~g}$ or more; (3) gestational age over 36 weeks; (4) the newborn was not treated with oxygen for longer than 30 minutes after birth; (5) the newborn did not need neonatal intensive care, parenteral feeding, or intravenous antibiotics; and (6) the newborn did not have any disorder that required substitution or supplementation.

\section{FEEDING INTERVENTION}

After birth, participating newborns were assigned randomly to receive either a supplementary standard whey protein dominant cows' milk formula, containing $11.1 \mathrm{~g}$ proteins $/ 100 \mathrm{~g}$ powder (Nutrilon Premium; Nutricia, Zoetermeer, the Netherlands), or a placebo formula. Both products were made available as a powder, and were packed in sachets that were reconstituted with $60 \mathrm{ml}$ of water. The placebo product designed for this study was based on a maltodextrin, glucose, and mineral solution emulsified with vegetable fats, visibly indistinguishable from infant formula. All ingredients of the placebo were free from cows' milk protein and the product was essentially protein free as assessed by the Kjeldahl method (detection limit $1 \mathrm{mg} / \mathrm{sach}$ t). The intervention feeding was given at least three times during the first three days after randomisation; each time one sachet or a part of a sachet was given supplementary to breast feeding or instead of one breast feed. The intervention feeding was discontinued if the newborn's condition deteriorated and could not be reversed by giving extra intervention feeding, water, glucose solution, or fennel seed water. Other reasons for discontinuation were hospital admission of the newborn with parenteral feeding or treatment with antibiotics, or if the mother decided not to continue. The mothers were encouraged to continue breast feeding without giving protein containing formula supplements for at least six weeks. Newborns who received formula milk, additionally or exclusively, remained in the study. The mothers did not receive any instructions or guidance about avoiding cows' milk and other potential allergens in their own diet during pregnancy and during the breast feeding period. The randomisation codes were computer generated, in equal proportions, in blocks of eight. The randomisation units were the children themselves. The intervention feeding was distributed to the recruiting midwives, packaged in sequentially numbered envelopes containing six sachets of cows' milk formula or six sachets of placebo, in agreement with the randomisation code. After verification of the inclusion criteria, the midwives assigned the next available number - that is the envelope that contained the intervention feeding and the corresponding forms. The randomisation number and the neonatal specifications were sent to the coordinating centre at the department of paediatrics of the Academic Medical Center of the University of Amsterdam. All empty and unused sachets were returned as a measure of compliance.

FOLLOW UP AND DATA COLLECTION

The participating newborns were followed up until the age of 2 years (fig 1). For the last child who entered the study, the follow up was completed in February 1996. Demographic characteristics, family history of allergic disorders,

Table 1 Characteristics of the study population

\begin{tabular}{|c|c|c|}
\hline Characteristic $(n=1533)$ & $\begin{array}{l}\text { Cows' milk } \\
(n=758)\end{array}$ & $\begin{array}{l}\text { Placebo } \\
(n=775)\end{array}$ \\
\hline \multicolumn{3}{|l|}{ Sex } \\
\hline Female $(n=753)$ & $48(365)$ & $50(388)$ \\
\hline Male $(\mathrm{n}=780)$ & $52(393)$ & $50(387)$ \\
\hline \multicolumn{3}{|l|}{ Place of delivery } \\
\hline At home $(\mathrm{n}=717)$ & $48(363)$ & $46(354)$ \\
\hline Hospital $(\mathrm{n}=816)$ & $52(395)$ & $54(421)$ \\
\hline \multicolumn{3}{|l|}{ Mother's age at birth (years) } \\
\hline$<25(\mathrm{n}=129)$ & $8(60)$ & $9(69)$ \\
\hline $25-29(\mathrm{n}=654)$ & $42(321)$ & $43(333)$ \\
\hline $30-34(\mathrm{n}=596)$ & $40(302)$ & $38(294)$ \\
\hline$\geqslant 35(\mathrm{n}=154)$ & $10(75)$ & $10(79)$ \\
\hline \multicolumn{3}{|l|}{ Maternal education * } \\
\hline Low $(n=203)$ & $13(100)$ & $13(103)$ \\
\hline Middle $(n=614)$ & $40(304)$ & $40(310)$ \\
\hline High $(n=714)$ & $47(353)$ & $47(361)$ \\
\hline Unknown $(\mathrm{n}=2)$ & $0(1)$ & $0(1)$ \\
\hline \multicolumn{3}{|l|}{ Number of siblings $†$} \\
\hline $0(\mathrm{n}=757)$ & $48(365)$ & $51(392)$ \\
\hline $1(\mathrm{n}=509)$ & $35(263)$ & $32(246)$ \\
\hline $2(\mathrm{n}=186)$ & $13(96)$ & $12(90)$ \\
\hline$\geqslant 3(n=51)$ & $3(20)$ & $4(31)$ \\
\hline Unknown, not $0(\mathrm{n}=30)$ & $2(14)$ & $2(16)$ \\
\hline \multicolumn{3}{|l|}{ Family risk of atopy } \\
\hline High $(\mathrm{n}=880)$ & $58(437)$ & $57(443)$ \\
\hline Low $(n=653)$ & $42(321)$ & $43(332)$ \\
\hline \multicolumn{3}{|l|}{ Pets at home } \\
\hline No $(n=623)$ & $43(323)$ & $39(300)$ \\
\hline Yes $(\mathrm{n}=910)$ & $57(435)$ & $61(475)$ \\
\hline \multicolumn{3}{|c|}{ Mother smoked during pregnancy } \\
\hline No $(n=1218)$ & $79(601)$ & $80(617)$ \\
\hline Yes $(n=295)$ & $20(150)$ & $19(145)$ \\
\hline Unknown $(\mathrm{n}=20)$ & $1(7)$ & $2(13)$ \\
\hline
\end{tabular}

Values are $\%(n)$.

^Low, no or primary education; middle, secondary education; high, higher professional and university education.

†Ranking was assessed at age 1 year but is corrected for changes during the first year. 
Table 2 Clinical outcome over two years, and laboratory and combined outcome in the first year for all randomised children (intention to treat analysis)

\begin{tabular}{|c|c|c|c|}
\hline Outcome & Cows'milk & Placebo & $\begin{array}{l}\text { Relative risk } \\
(95 \% \mathrm{CI})\end{array}$ \\
\hline \multicolumn{4}{|c|}{ Clinical outcome } \\
\hline \multicolumn{4}{|c|}{ Atopic disease in the first year $(n=1482)$} \\
\hline Obvious $(\mathrm{n}=143)$ & $10.0(73 / 730)$ & $9.3(70 / 752)$ & $1.07(0.79-1.47)^{\star}$ \\
\hline Possible $(\mathrm{n}=348)$ & $23.4(171 / 730)$ & $23.5(177 / 752)$ & $1.02(0.88-1.17) \dagger$ \\
\hline No $(n=991)$ & $66.6(486 / 730)$ & $67.2(505 / 752)$ & Reference \\
\hline \multicolumn{4}{|c|}{ Atopic disease in the second year $(n=1220)$} \\
\hline Obvious $(\mathrm{n}=121)$ & $9.6(57 / 594)$ & $10.2(64 / 626)$ & $0.94(0.67-1.32)^{\star}$ \\
\hline Possible $(n=254)$ & $19.9(118 / 594)$ & $21.7(136 / 626)$ & $0.92(0.78-1.09) \dagger$ \\
\hline No $(\mathrm{n}=845)$ & $70.5(419 / 594)$ & $68.1(426 / 626)$ & Reference \\
\hline \multicolumn{4}{|c|}{ Maximal outcome in the first two years $(n=1482)$} \\
\hline Obvious $(\mathrm{n}=180)$ & $11.9(87 / 730)$ & $12.4(93 / 752)$ & $0.96(0.73-1.27)^{\star}$ \\
\hline Possible $(n=413)$ & $27.8(203 / 730)$ & $27.9(210 / 752)$ & $0.99(0.87-1.12) \dagger$ \\
\hline No $(\mathrm{n}=889)$ & $60.3(440 / 730)$ & $59.7(449 / 752)$ & Reference \\
\hline \multicolumn{4}{|l|}{ Laboratory outcome, $R A S T \ddagger$} \\
\hline Any RAST $(\mathrm{n}=1434)$ & $9.4(66 / 702)$ & $7.9(58 / 732)$ & $1.19(0.85-1.66)$ \\
\hline Cows' milk $(\mathrm{n}=1434)$ & $5.8(41 / 702)$ & $4.1(30 / 732)$ & $1.43(0.90-2.26)$ \\
\hline Hens' eggs $(n=1434)$ & $4.4(31 / 702)$ & $4.9(36 / 732)$ & $0.90(0.56-1.44)$ \\
\hline House dust mite $(\mathrm{n}=1425)$ & $0.3(2 / 700)$ & $0.1(1 / 725)$ & $2.07(0.19-22.79)$ \\
\hline Cat dander $(n=1422)$ & $1.7(12 / 699)$ & $1.5(11 / 723)$ & $1.13(0.50-2.54)$ \\
\hline Dog dander $(\mathrm{n}=1422)$ & $0.7(5 / 699)$ & $0.8(6 / 723)$ & $0.86(0.26-2.81)$ \\
\hline
\end{tabular}

Combined outcome

Atopy $(\mathrm{n}=1428)$

Obvious $(\mathrm{n}=172)$

Possible $(n=356)$

No $(\mathrm{n}=900)$

$\begin{array}{lll}11.7(82 / 702) & 12.4(90 / 726) & 0.94(0.71-1.25)^{\star} \\ 26.8(188 / 702) & 23.1(168 / 726) & 1.08(0.95-1.24) \dagger\end{array}$

$61.5(432 / 702) \quad 64.5(468 / 726) \quad$ Reference

Values are $\%(\mathrm{n} /$ total $)$

$\star$ Obvious versus possible and no. $†$ Any (obvious and possible) versus no. $\ddagger$ Positive $(\geqslant 2+)$.

and diet habits of the mother during pregnancy and during the breast feeding period were recorded before birth and at ages 1 and 2 years. Breast feeding and volumes of intervention feeding and other supplements, if given, were recorded daily during the first 2 weeks of life. Feeding from the third until the sixth week was recorded weekly. From 6 weeks until the first birthday, parents documented any food other than breast feeding, with the date of introduction and the child's reaction to the food. At 1 year (physical examination) and at 2 years of age (questionnaire), the total daily amount of food intake was recorded, as were adverse reactions to foods, reasons for changing feeding habits and, if applicable, elimination and re-introduction of certain food products. The health status of the child was assessed by questionnaires at 6 weeks, 6 months, 1 year, and 2 years of age. From the sixth week until the age of 2 years, a weekly symptom score was recorded by the parents. Symptoms included eczema, itching, and possible allergic symptoms of the airways and gastrointestinal tract. The children were seen by two of the investigators (MdJ and VS) at 1 year of age and were

Table 3 Clinical outcome over two years for all randomised children who received at least three intervention feeds and exclusively breast feeding for six weeks (per protocol analysis)

\begin{tabular}{|c|c|c|c|}
\hline Clinical outcome & Cows'milk & Placebo & Relative risk $(95 \% \mathrm{CI})$ \\
\hline \multicolumn{4}{|c|}{ Atopic disease in the first year $(\mathrm{n}=768)$} \\
\hline Obvious $(\mathrm{n}=72)$ & $9.8(35 / 358)$ & $9.0(37 / 410)$ & $1.08(0.70-1.68)^{\star}$ \\
\hline Possible $(\mathrm{n}=155)$ & $19.6(70 / 358)$ & $20.7(85 / 410)$ & $0.99(0.79-1.23) \dagger$ \\
\hline No $(n=541)$ & $70.7(253 / 358)$ & $70.2(288 / 410)$ & Reference \\
\hline \multicolumn{4}{|c|}{ Atopic disease in the second year $(n=639)$} \\
\hline Obvious $(n=66)$ & $8.9(26 / 291)$ & $11.5(40 / 348)$ & $0.78(0.49-1.24)^{\star}$ \\
\hline Possible $(n=129)$ & $19.6(57 / 291)$ & $20.7(72 / 348)$ & $0.89(0.70-1.12) \dagger$ \\
\hline No $(\mathrm{n}=444)$ & $71.5(208 / 291)$ & $67.8(236 / 348)$ & Reference \\
\hline \multicolumn{4}{|c|}{ Maximal outcome in the first two years $(n=768)$} \\
\hline Obvious $(\mathrm{n}=89)$ & $11.5(41 / 358)$ & $11.7(48 / 410)$ & $0.98(0.66-1.45)^{\star}$ \\
\hline Possible $(n=201)$ & $26.3(94 / 358)$ & $26.1(107 / 410)$ & $1.00(0.83-1.20) \dagger$ \\
\hline No $(n=478)$ & $62.3(223 / 358)$ & $62.2(255 / 410)$ & Reference \\
\hline
\end{tabular}

Values are $\%$ (n/total).

*Obvious versus possible and no. †Any (obvious and possible) versus no. examined for the presence and severity of atopic eczema, (rhino) conjunctivitis, wheezing and other clinical signs of allergy. Also, an intravenous blood sample was taken for the assessment of specific IgE by the radioallergosorbent test (RAST) against cows' milk protein, hen's egg protein, house dust mite, cat dander, and dog dander. These laboratory tests were expressed as negative (normal) or ranged from $1+$ (dubious) to $5+$ (strongly positive).

\section{OUTCOME DEFINITIONS AND DATA ANALYSIS}

The primary outcome was atopic disease in the following categories: obvious atopic disease, possible atopic disease, and no atopic disease (clinical outcome). The outcome was assessed over three time periods: in the first year, in the second year, and in the first 2 years of life taken together (maximal outcome). We put children into the category of obvious atopic disease if any of the following had occurred: (1) a documented episode of an acute, anaphylactic reaction to an allergen; (2) at least one episode of atopic eczema or dermatitis, as defined by Hanifin and Rajka ; (3) an episode of nonpurulent rhinitis or conjunctivitis or both during four weeks, or four episodes in a year, possibly related to a specific allergen; (4) at least three episodes of asthma or wheezing in a year; (5) an episode of at least three of the following gastrointestinal symptoms: periods of nausea or vomiting, flatus and distended abdomen, or periods of diarrhoea related to a specific kind of food, disappearing after elimination of the suspected food from the infant's diet, and recurring upon re-introduction of this food; or (6) children with any of these conditions, diagnosed by their general practitioner or paediatrician, and successfully treated.

Children who fulfilled at least one of the following criteria were put into the category of possible atopic disease: (1) presence of some but not all criteria for atopic dermatitis; (2) non-purulent rhinitis or conjunctivitis only two or three times a year; (3) respiratory symptoms suggesting asthma or wheezing once or twice a year; or (4) two of the mentioned gastrointestinal symptoms related to food intake and suggestive of an allergic reaction, which reacted well to elimination of the suspected food, but without re-introduction of this food. The remaining children were put into the category of no atopic disease.

The clinical outcome was determined for the first and second year of life by the two investigators (MdJ and VS), independent of one another and blinded for the intervention. When differences were found, a consensus was reached after mutual consultation. Classification was derived from the questionnaires, weekly symptom scores, feeding records and, for the first year, from the physical examination. The maximal outcome was defined as the highest classification in the first 2 years, or the classification in the first year if the follow up in the second year was not completed.

The secondary outcome (laboratory outcome) was any positive RAST at 1 year of age. 
Secondary outcome was considered to be positive if any of the five RASTs was positive $(2+$ or more).

Finally, a combined outcome of clinical features and laboratory outcome was conducted at 1 year of age. Obvious atopy was defined as clinically obvious atopic disease regardless of the outcome of the RAST, or as possible atopic disease with positive RAST. Possible atopy was defined as clinically possible atopic disease without positive RAST, or as no clinical atopic disease with any RAST positive. No atopy was defined as a negative clinical outcome and a negative laboratory outcome.

We performed two analyses: an intention to treat analysis and a per protocol analysis. The primary analysis was the intention to treat analysis for the clinical, the laboratory, and the combined outcome. Primary analysis was carried out for the whole study population, irrespective of the actual feeding regimen. Per protocol analysis, performed for the clinical outcome, was restricted to those children who received at least three intervention feeds and who were breast fed exclusively for six weeks. Finally, we analysed the clinical outcome for the children who had a high or a low family risk for atopy. A high risk was defined as at least one parent or sibling having atopic symptoms. We calculated the relative risks with corresponding $95 \%$ confidence intervals $(\mathrm{CI})$.

\section{Results}

Over the two year period, 1693 informed consent forms were received with the assistance of 70 midwives. After birth, 1533 children were randomised for the study (fig 2). Reasons for exclusion $(n=160)$ were: low birth weight $(n=28)$, neonatal antibiotics $(n=25)$, oxygen after birth for more than 30 minutes $(n=16)$ or both neonatal antibiotics and oxygen $(n=7)$, and other reasons $(n=84)$, such as unawareness of the study because the midwife was off duty, lack of cooperation of a hospital, mother's decision to feed formula milk. The intervention feeding was well tolerated and no escape from intervention because of deterioration was required. One child died of meningitis at the age of 4 months.

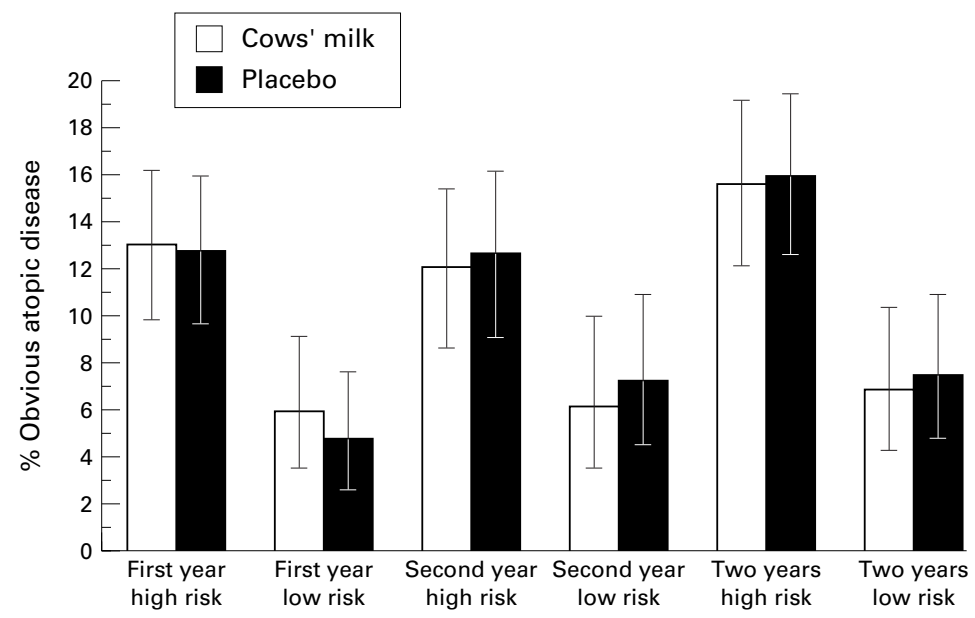

Figure 3 Percentage obvious atopic disease and 95\% confidence intervals in the first 2 years of life, stratified for family risk of atopy.
Ninety seven per cent of the children were seen at 1 year of age, $80 \%$ completed the follow up to the age of 2 years. No substantial differences in baseline characteristics were found between the intervention groups (table 1). Intervention feeding was given in agreement with the study protocol to $93 \%$ of the children $(n=1422)$. Mean volumes in the first 4 days of life were $22 \mathrm{ml}, 43 \mathrm{ml}, 40 \mathrm{ml}$, and $15 \mathrm{ml}$, respectively; the median amount in the first week was $120 \mathrm{ml}$, range, $10-360 \mathrm{ml}$, for both intervention groups. The criterion of exclusively breast feeding for six weeks was fulfilled by $54 \%$ of the children $(\mathrm{n}=824)$. As shown in fig 2,777 children $(51 \%)$ were included for the per protocol analysis.

Clinically obvious atopic disease in the first year of life was diagnosed in 73 children $(10.0 \%)$ of the cows' milk intervention group $(n=730)$, and in 70 children $(9.3 \%)$ of the placebo group $(n=752)$. This difference was not significant (relative risk, 1.07; 95\% CI, 0.79 to 1.47 ). In the second year of life, we found no differences in the incidence of atopic disease between the two study groups: $9.6 \%$ (cows' milk) v 10.2\% (placebo); relative risk, 0.94 ; $95 \%$ CI, 0.67 to 1.32 . The maximal outcome in the first 2 years of life was $11.9 \%$ (cows' milk) $v 12.4 \%$ (placebo) with obvious atopic disease. Laboratory outcome (any RAST) at 1 year of age showed $9.4 \%$ of the children with a positive outcome in the cows' milk group and $7.9 \%$ in the placebo group (relative risk, $1.19 ; 95 \%$ CI, 0.85 to 1.66 ). The combined outcome at 1 year of age showed obvious atopy in $11.7 \%$ (cows' milk) $v 12.4 \%$ (placebo) of children (table 2). In almost $80 \%$ of the children with obvious atopic disease, the diagnosis was based on atopic dermatitis or gastrointestinal disease caused by food allergy. Respiratory symptoms were the major reasons for classifying possible atopic disease.

Per protocol analysis showed results similar to those of the intention to treat analysis (table 3).

The analysis by family risk of atopy showed a doubled incidence of obvious atopic disease in the high risk children, but the intervention did not have any effect (fig 3). The $95 \%$ CI for the differences between the high risk and the low risk groups on cows' milk were 3\% to $11 \%$ for the first year, $1.4 \%$ to $10.5 \%$ for the second year, and $4.3 \%$ to $13.2 \%$ for the first 2 years together.

\section{Discussion}

Keeping in mind the standards suggested by Kramer in his review, we designed this double blind, randomised study with blind assessment of the outcome. ${ }^{3}$ Our response was high, 97\% at 1 year of age and $80 \%$ at 2 years. The number of high risk families was higher than in the general Dutch population. This probably reflects an increased interest of mothers in high risk families to participate in a study like ours and a strong motivation to breast feed their child.

In our study, breast fed children who received cows' milk supplementation during the first 3 days of life had the same risk of a clinical or serological expression of atopic 
disease as those who received a placebo formula. Therefore, we could not confirm the findings of Høst et al that formula feeding during the first 3 days increases the risk of atopic disease. ${ }^{1}$ This discrepancy could be because their cohort was analysed retrospectively. In addition, we could not confirm the results of Lindfors and Enocksson, who found a protective effect of formula feeding before breast feeding. ${ }^{2}$ Their study involved "small for dates" children, whereas we followed full term, healthy children. During the period of our study, Schmitz et al presented the preliminary results of a small, randomised study. ${ }^{5}$ They used a different intervention regimen, but the results are similar to our findings. The authors compared supplementation with an adapted formula and a partially hydrolysed formula in 256 normal breast fed infants and found no significant difference in the prevalence of clinical symptoms and IgE responses. Oldæus et al evaluated the prophylactic effect of extensively and partially hydrolysed formula on the development of atopic symptoms in high risk infants. ${ }^{6}$ Their findings support an allergy preventive effect only of the extensively hydrolysed formula. However, they did not investigate brief neonatal supplementation, but evaluated a nine month period of dietary restrictions of mother and child, with formula intervention from the start of weaning.

The median volume of formula feeding in our study was $120 \mathrm{ml}$. This is considerably more than the estimated minimum amount required to induce sensitisation, because children can be sensitised by breast feeding exclusively. ${ }^{78}$
Formula milk is often given before breast feeding is established and is sometimes even indicated. Our results suggest that strict feeding regimens are not needed in the neonatal period, at least with regard to the development of early onset allergy, as assessed by the development of atopic dermatitis and gastrointestinal disease caused by food allergy. Two years might be too short a study period to draw a conclusion about the risk or benefit towards asthma. Therefore, a second study is underway, focusing on respiratory symptoms at the age of 5 years, as a next step in dispelling the fears related to the concept of "the dangerous bottle".

This study was funded by Nutricia Nederland BV, Zoetermeer, the Netherlands. The support from Nutricia Research in developing the placebo product and making the blinded and packaged intervention products available is greatly appreciated. The authors thank the local paediatricians who were affiliated with the initiation of the study and all midwives who with the initiation of the study and all midn
participated and whose cooperation was essential.

1 Høst A, Husby S, Østerballe O. A prospective study of cows' milk allergy in exclusively breast fed infants. Acta Pcediatr milk allergy in exclusive

2 Lindfors A, Enocksson E. Development of atopic disease Lindfors A, Enocksson E. Development of atopic disease
after early administration of cows' milk formula. Allergy 988;43:11-16.

3 Kramer MS. Does breast feeding help protect against atopic disease? Biology, methodology and a golden jubilee of controversy. F Pediatr 1988;112:181-90.

4 Hanifin JM, Rajka G. Diagnostic features of atopic dermatitis. Acta Dermatol Venereol Suppl (Stockh) 1980;S92:44-7.

5 Schmitz J, Digeon B, Chastang C, et al. Effects of brief early exposure to partially hydrolyzed and whole cows' milk proteins. F Pediatr 1992;121:S85-9.

6 Oldæus G, Anjou K, Björkstén B, Moran JR, Kjellman N-IM. Extensively and partially hydrolysed infant formulas for allergy prophylaxis. Arch Dis Child 1997;77:4-10.

7 Høst A. Cows' milk protein allergy and intolerance in Høst A. Cows milk protein allergy and intolerance
infancy. Pediatr Allergy Immunol 1994;5(suppl 5):5-36.

8 Strobel S. V. Mechanisms of tolerance and sensitization in the intestine and other organs of the body. Allergy 1995;50(suppl 20): 18-25. 